P. Gerlinger, P. Stoll, M. Kindler, F. Schneider, M. Aigner, Numerical Investigation of Mixing and Combustion Enhancement in Supersonic Combustors by Strut Induced Streamwise Vorticity, Aerosp. Sci. Technol. 12, (2008) 159-168.

The original publication is available at www.elsevier.com

http://dx.doi.org10.1016/j.ast.2007.04.003 


\title{
Numerical Investigation of Mixing and Combustion Enhancement in Supersonic Combustors by Strut Induced Streamwise Vorticity
}

\author{
Peter Gerlinger ${ }^{\mathrm{a}, 1}$ \\ ${ }^{a}$ Institute of Aerospace Combustion Technology (IVLR), University of Stuttgart, \\ Pfaffenwaldring 38-40, 70569 Stuttgart, Germany \\ Peter Stoll ${ }^{\mathrm{b}}$ \\ ${ }^{\mathrm{b}}$ Institute of Aerospace Thermodynamics (ITLR), University of Stuttgart, \\ Pfaffenwaldring 31, 70569 Stuttgart, Germany ${ }^{2}$ \\ Markus Kindler, Fernando Schneider, Manfred Aigner ${ }^{c}$ \\ ${ }^{\mathrm{c}}$ Institute of Combustion Technology, DLR Stuttgart, Pfaffenwaldring 38-40, 70569 \\ Stuttgart, Germany
}

\begin{abstract}
A numerical study of mixing and combustion enhancement has been performed for a Mach 2 model scramjet (supersonic combustion ramjet) combustor. Fuel (hydrogen) is injected at supersonic speed through the rear of a lobed strut located at the channel symmetry axis. The shape of the strut is chosen in a way to produce strong streamwise vorticity and thus to enhance the hydrogen/air mixing. Strength and size of the vortices are defined by the strut geometry and may be modified. It will be shown that in comparison to planar struts the mixing efficiency is strongly improved. On the other hand, the induced vortices cause an increase in entropy and larger losses in total pressure. Different planar and lobed strut injectors are investigated numerically and a comparison with experimental data is given for cold supersonic mixing. Based on this study a numerical investigation of flame stabilization and fuel burnout is performed where two stable modes of combustion are identified. They are associated with attached or detached flames depending on the chosen inflow conditions. In both cases subsonic regions at the channel symmetry axis are responsible for flame holding. If the combustor geometry is chosen in a favourable way these subsonic zones may be kept small. Moreover, the flames are away from solid walls thus minimizing the wall heat load.
\end{abstract}

Preprint submitted to Elsevier Science

18 April 2007 
Key words: Streamwise vorticity, mixing enhancement, supersonic combustor, scramjet, hydrogen/air combustion, lobed strut injector

PACS:

\section{Introduction}

Due to the extremely short residence time of the air in supersonic combustors, an efficient (rapid and with small losses in total pressure) fuel/air mixing is hard to achieve. Nevertheless this is an important issue to keep the combustor length short and to reduce the skin friction drag. In supersonic flows a rapid fuel/air mixing additionally suffers from inherently low mixing rates due to compressibility effects at high convective Mach numbers [1,2]. There are mainly two concepts for fuel injection in supersonic combustors:

- wall injectors, where hydrogen is injected through the wall [3-5] (normal or oblique to the main flow) or by ramps [6-8] mounted to the wall,

- and strut injectors [1,9-11], which are located at the channel axis and directly inject the fuel into the core of the air stream.

In some cases both types of injectors approach each other, e.g. if a ramp injector extends over most of the channel height [8]. A good near field mixing can be achieved by wall injection. On the other hand transverse injection systems cause a significant blockage of the flow resulting in irreversibilities due to shock waves and thrust losses [12]. Another concern is that the penetration of the fuel jet may be insufficient for real size combustors. Wall injectors have the advantage of being easy to manufacture, easy to cool, and, in case of staged injections, they cause no losses in total pressure if they are switched off. The last point is in contrast to ramp or strut injectors which may not be removed from the flow field if no hydrogen is injected. Moreover, the injected hydrogen usually acts as a coolant for the strut. This has to be kept in mind if the hydrogen mass flux is reduced. An alternative to physical ramp injectors are aeroramps [13] which have a similar physical behaviour but lower pressure losses [14]. Aeroramps are multi-hole transverse injectors which induce pairs of counter-rotating vortices to improve mixing and fuel penetration.

If strut injectors are used, usually all or most of the fuel is injected in main flow direction. This is possible without the induction of strong shock waves. Moreover, additional momentum is added by parallel fuel injection increasing the engine thrust. This may become important at high flight Mach numbers (10-

\footnotetext{
Email address: peter.gerlinger@dlr.de (Peter Gerlinger ).

1 Corresponding author

2 former member
} 
15) [15]. Due to the limited mixing capabilities of parallel high speed streams, techniques for mixing enhancement are required. This can be achieved either by the use of shock waves $[16,17]$ or by creation of streamwise vorticity. Streamwise vortices may be induced by a favourable chosen strut geometry. Several such concepts have been presented in the past $[9,11,18,19]$. The subject of this paper is a lobed strut injector which has been investigated experimentally at the ITLR (Institute of Aerospace Thermodynamics, University of Stuttgart) and numerically by the IVLR (Institute of Aerospace Combustion Technology, University of Stuttgart) and by DLR-VT (Institute of Combustion Technology, DLR Stuttgart). For these injectors detailed information about species distributions and the influence of streamwise vorticity on the hydrogen mixing behaviour will be given. Hydrogen should be injected in such a way that a good mixing is achieved over a short length resulting in a homogeneous temperature distribution. Local temperature peaks have to be avoided as to keep dissociation losses and nitrogen oxides low.

An important issue at low flight Mach numbers of a scramjet is autoignition. Due to relatively low air static temperatures this may become a problem for axial strut injectors which only induce weak shock waves and small recirculation zones downstream of the strut. Thus the advantage of avoiding normal shock waves (as in case of transverse injection) may cause problems for a stable ignition. Four different modes of combustion may be distinguished for strut injectors:

- A stable combustion where the flame is (attached) anchored directly downstream of the strut,

- a stable combustion with a lifted flame which may be stabilized by shock waves and/or a subsonic zone,

- blow-off of the flame due to unfavourable thermodynamic conditions or bad mixing,

- thermal choking where the heat release is too high, the flame is moving upstream, and a normal shock causes subsonic flow in the combustor. The subsequent high temperatures usually will cause damage to the strut.

The last two modes have to be avoided and the second one usually reacts sensitive to changes of the inflow conditions. At low flight Mach numbers (Ma $\approx 7-8)$ and therefore low total temperatures $\left(T_{0}=1200-1700 \mathrm{~K}\right)$ attached or detached flames are possible [20]. In both cases combustion (at least partially) takes place in subsonic regions. Pure supersonic combustion is obtained at higher flight Mach numbers $(M a>8)$. The present paper deals with combustor inlet conditions which correspond to the low flight Mach number range. 


\section{Numerical scheme}

The following numerical investigations are performed using the inhouse TAS-

COM3D (Turbulent All Speed Combustion Multigrid) solver [21-23]. This code has been developed over more than a decade for the simulation of supersonic combustion. It is validated with a large number of different supersonic test cases with $[21,22,18,24,25]$ and without $[26,23,18]$ combustion. TASCOM3D solves the full compressible Navier-Stokes, turbulence and species transport equations. Combustion is treated by finite-rate chemistry. The unsteady form of the governing equations is integrated in time using an implicit finite-volume LU-SGS (Lower-Upper Symmetric Gauß-Seidel) algorithm $[27,28]$. Beside the source term Jacobian and inviscid flux Jacobians, simplified viscous flux Jacobians based on the thin-layer Navier-Stokes equations are included in the implicit part of the numerical solver. The discretization is second order in space and first or second order in time. To allow simulations of compressible and incompressible flows an all-Mach-number preconditioning is included. The code is based on block structured grids and is fully vectorized and parallelized using MPI [29]. All results presented in this paper are obtained on parallel systems by domain decomposition. For turbulence closure a low-Reynolds-number $q-\omega[30](q=\sqrt{k}, k$ is the turbulent kinetic energy, and $\omega=\epsilon / k, \epsilon$ is the dissipation rate of $k$ ) turbulence model is employed which requires very fine grids near solid walls. To achieve a good accuracy $y^{+}$ values below one should be reached at all near wall cell centres. The turbulent Prandtl and Schmidt numbers are chosen to be 0.7 . For the modeling of hydrogen combustion the 9-species, 20-step reaction mechanism of Jachimowski [31] is used excluding the nitrogen reactions.

\section{Model scramjet combustor}

The numerically simulated struts and combustor geometries are based on experimental investigations performed at the supersonic combustor test facility of the ITLR (University of Stuttgart) [32]. For cold supersonic mixing experimental results are available for planar and lobed strut injectors. Figure 1 shows a sketch of the planar and lobed struts. The combustor geometry and the position of the strut is given in Fig. 2. Wall pressures and species distributions $\left(\mathrm{H}_{2}, \mathrm{O}_{2}\right.$, and $\left.\mathrm{N}_{2}\right)$ have been measured and this data is used for code validation. 


\subsection{Combustor geometry}

The geometry of the model scramjet combustor plotted in Fig. 2 corresponds to the area covered by the computational grid. The simulation starts with sonic conditions at the air nozzle throat. After expansion in the planar nozzle a flow Mach number of 2.1 is reached. Downstream of the nozzle the channel has a constant cross section of $36 \mathrm{x} 40 \mathrm{~mm}$ (channel height $\mathrm{x}$ depth). In case of pure mixing the channel cross section is kept constant while for combustion a diverging combustor geometry is used. All simulations are performed in two steps: A first two dimensional inert air simulation up to the middle of the strut (to deliver inflow values) and a second reactive three-dimensional simulation of the remaining combustor.

\subsection{Strut injector}

Hydrogen is injected in axial direction through the rear of the strut directly into the core of the air flow. A nozzle inside the strut accelerates the hydrogen up to an exit Mach number of about two. All struts investigated have a length of $80 \mathrm{~mm}$, a height of $6 \mathrm{~mm}$, and a width of $40 \mathrm{~mm}$. They have been used experimentally $[32,18]$ and have proven to work reliably even in long time combustion experiments [34]. The wall thickness at the end of the strut is $0.35 \mathrm{~mm}$ and the thickness of the injected hydrogen jet is $0.6 \mathrm{~mm}$. Due to the small thickness of the hydrogen jet and its low momentum, velocity differences between hydrogen and air disappear quickly.

The manner in which the shape of the lobed strut produces streamwise vorticity is explained in Fig. 3 by a simplified sketch for one strut segment. At the tip of the strut a shock wave is formed which is followed by two expansion fans at the middle of the strut. Due to the different changes in flow direction, these expansion fans have different strengths. As a result the static pressures after expansion differ between the front and the back of the plotted segment (in depth). This pressure difference causes a secondary flow in crosstream direction. At the bottom side of the strut the same process takes place in opposite direction where the induced secondary flow has the same axis and direction of rotation. Both flows in crosstream direction (above and below the strut) unite and form a vortice at the struts' end. In this way pairs of counter-rotating vortices are induced (five vortices in case of the investigated combustor) into which the hydrogen is injected. The following numerical simulations will show, that the vorticity production and mixing enhancement by the lobed structure works well both in non-reactive and reactive flow. Figure 4 shows streamlines in a view from the channel exit in upstream direction. These streamlines are 
obtained from a combustion simulation and start at the end of the strut. Over the plotted length of about $450 \mathrm{~mm}$ the lobed injector causes a rotation of the streamlines of more than $180^{\circ}$. Similar results are obtained for non-reactive flows. Strength and size of the vortices are defined by the strut geometry and thus allow a controlled induction of circulation. However, modification of the strut geometries are not the topic of this paper.

In the following numerical study three lobed strut injectors are compared which have the same geometry but differ in the areas of their hydrogen nozzles. The black areas plotted in Fig. 5 indicate the nozzle exit areas at the rears of the struts where the hydrogen is injected. All remaining parts of the struts are identical. By varying the hydrogen injection areas the location of fuel injection with respect to the induced vortices is changed. Moreover the area of interfacial contact between the hydrogen jet and the surrounding air is modified. Downstream of the strut this contact area is increased due to deformation caused by the vortices. As to take advantage of this effect, injection should take place away from the axises of rotation. In the subsequent studies the hydrogen injection conditions and the air inflow conditions are kept constant both for the non-reacting and reacting test cases, respectively. Due to the constant fuel supply and varying nozzle areas the hydrogen mass flow rates differ for the different struts.

\subsection{Performance parameters}

To evaluate the mixing and combustion process along the channel length performance parameters are needed which are calculated by taking averages over the respective channel cross sections. The mixing efficiency $\left(0 \leq \eta_{\operatorname{mix}} \leq 1\right)$

$$
\begin{aligned}
& \eta_{\text {mix }}(x) \equiv \frac{\int_{A} \alpha \rho u Y_{\mathrm{H}_{2}} d A}{\int_{A} \rho u Y_{\mathrm{H}_{2}} d A}=\frac{\int_{A} \alpha \rho u Y_{\mathrm{H}_{2}} d A}{\dot{m}_{\mathrm{H}_{2}}(x)}, \\
& \text { with } \quad \alpha=\left\{\begin{aligned}
1 / \Phi: & \Phi \geq 1 \\
1: & \Phi<1
\end{aligned}\right.
\end{aligned}
$$

is defined as the fraction of hydrogen mass flux that could be burned (at the given state of mixing in case of infinitely fast chemistry) in relation to the total hydrogen mass flux. In Eq. (1) $\Phi$ is the equivalence ratio, $A$ indicates the channel cross section, $\rho$ the density, $u$ the normal velocity, $Y_{\mathrm{H}_{2}}$ the mass fraction of hydrogen, and $\dot{m}_{\mathrm{H}_{2}}(x)$ is the total mass flux of unburned hydrogen through the channel cross section at position $x$. In case of non-reactive flow $\dot{m}_{\mathrm{H}_{2}}(x)=\int_{A} \rho u Y_{\mathrm{H}_{2}} d A$ is constant downstream of the injector and $\eta_{\text {mix }}(x)$ is a non-decreasing function. For $\eta_{\text {mix }}=1$ a stoichiometric or fuel lean mixture 
is reached where enough oxygen is available to completely burn all hydrogen. However, $\eta_{\operatorname{mix}}(x)$ is also an important parameter in case of combustion, where it always relates (at any $x$-position) to the remaining (unburned) hydrogen mass flux. Again $\eta_{\text {mix }}$ covers the range $0 \leq \eta_{\text {mix }} \leq 1$ but now it may decrease due to combustion. As in non-reactive flow it indicates which part of the of unburned hydrogen is mixed with oxygen in a way that it could react under favourable thermodynamic conditions. In case of incomplete combustion $\eta_{\text {mix }}$ clarifies if there is a problem of mixing or of chemical kinetics.

To evaluate the degree of burning the normalized cross section averaged hydrogen mass flux or similarly the burning efficiency $\left(0 \leq \eta_{\text {burn }} \leq 1\right)[35]$

$$
\eta_{\text {burn }}(x) \equiv 1-\frac{\left.\int_{A} \rho u Y_{\mathrm{H}_{2}} d A\right|_{\text {reacting }}}{\left.\int_{A} \rho u Y_{\mathrm{H}_{2}} d A\right|_{\text {non-reacting }}}=1-\frac{\dot{m}_{\mathrm{H}_{2}}(x)}{\dot{m}_{\mathrm{H}_{2, \text { injected }}}}
$$

is used. It shows for every $x$-location which part of the injected hydrogen mass flux is already burned.

As a last performance parameter the loss in total pressure is considered. Losses in total pressure are directly related to an increase in entropy and thrust losses. The normalized mass flux averaged total pressure is calculated for every channel cross section by

$$
\bar{p}_{t} \equiv \frac{1}{\dot{m}} \int_{A} p_{t} \rho u d A
$$

where $p_{t}$ is the total pressure (calculated from the local Mach number and local static pressure) and $\dot{m}$ is the total mass flux through the channel cross section. Losses in total pressure are caused by shock waves, the mixing process, and viscous effects, e.g. in boundary layers.

\section{Mixing investigation}

Experimental data of cold (non-reacting) hydrogen air mixing are available for the planar strut plotted in Fig. 1 and the lobed strut I (top sketch of Fig. $5)$, where the hydrogen nozzle area extends over the complete lobed structure at the end of the strut. The inflow conditions for air and hydrogen are summarized in Table I. They are in accordance with the inflow conditions in the experiments. Besides the two struts investigated experimentally, an additional simulation is performed for strut II (middle sketch of Fig. 5) because the nozzle area of this strut is nearly identical to that of the planar strut. 
The following simulations take advantage of all possible symmetries of the flow field (point and line symmetries) in order to reduce the number of necessary grid points. While the lower and upper channel walls are considered, symmetry conditions are assumed at the front and back sides of the computational domain. The grid is strongly refined at all near wall regions and in the mixing zone downstream of the injector. In total the computational grid consists of 2.2 million volumes. Nevertheless, there still are some minor grid dependencies which mainly result in smeared shock waves after some wall reflections. However, no visible grid influence on the the species distributions has been observed.

Figure 6 shows calculated and measured distributions of hydrogen molar fractions for channel cross sections located at $x=25,50,100$, and $150 \mathrm{~mm}(x=0$ is at the end of the strut). For every cross section results of the simulation are plotted on the left and experimental results on the right side. The hydrogen distributions show the effect of the vortices induced by the strut. The lobed S-shaped structure of the hydrogen jet directly downstream of the injector is deformed by the circulation of the flow field. The induced vortices have sizes in the order of half the channel height. They improve the macro-mixing by increasing the interfacial contact area between fuel and air and by production of large scale turbulence. Due to the energy transfer from large to small scales this also results in an increased micromixing which is important for combustion. Even if not used in the present case, mixing may be further improved by the production of turbulence of intermediate scales, e.g. by mounting small bodies at the end of the strut. As may be seen from the last distribution of Fig. 6 (corresponding to $x=150 \mathrm{~mm}$ ), the highest hydrogen concentrations remain near the axises of rotation (at $z=0 \mathrm{~mm}$ and $y=-8 \mathrm{~mm}$ or $y=0$ $\mathrm{mm}$, respectively). Due to the torsion of the S-shaped structure, the hydrogen injected from the vertical parts of the strut is not able to mix completely with the surrounding oxygen. Such fuel rich regions should be avoided. Figure 7 shows corresponding one dimensional profiles of hydrogen molar fractions along the $z$-axis for $y=0$ at the positions $x=3,50,100$, and $150 \mathrm{~mm}$ (see Fig. 1 for a definition of the different coordinate directions). In case of the lobed strut vorticity forces the hydrogen to move away from the channel axis in direction to the upper and lower walls. This becomes evident if the growth of the mixing layer thickness is considered. The mixing layer thickness (for every $x$-position) here is defined as the distance in $z$-direction between the transverse locations where the molar fractions of hydrogen are 0.05. While the numerically obtained mixing layer thickness $150 \mathrm{~mm}$ downstream of the injector is about $6.8 \mathrm{~mm}$ for the planar strut [33] it is more than $18 \mathrm{~mm}$ for the lobed strut.

Finally Fig. 8 compares calculated mixing efficiencies of the lobed strut II and the planar strut (their injected hydrogen mass fluxes and stoichiometric ratios are nearly identical). While it takes about $300 \mathrm{~mm}$ for the planar strut 
to achieve a perfect mixing, only $80 \mathrm{~mm}$ are required for strut II. The better mixing allows a shorter combustor length and thus a reduction in weight. Moreover, the losses in total pressure to reach $\eta_{\text {mix }}=1$ are about $15 \%$ smaller for the lobed strut. It also has to be mentioned that the fast mixing achieved in this study is also due to the understoichiometric ratio at which hydrogen is injected ( $\Phi=0.28$ for strut I and 0.19 for strut II, respectively). In case of hot air $\Phi$ increases because of lower air densities but it still remains understoichiometric. Approaching a global stoichiometric ratio the length to achieve a perfect mixing increases. Nevertheless, the improvements achieved by inducing streamwise vorticity remain.

\section{Combustion investigation}

The following numerical study is performed for combustor inlet conditions which correspond to low flight Mach numbers $(7-8)$ of a scramjet. The air inflow temperature at the nozzle throat is chosen to be $1300 \mathrm{~K}$ corresponding to a total temperature of $1560 \mathrm{~K}$. After expansion of the air a Mach number of 2.1 is reached in the combustor. The static pressure and temperature in front of the strut are 0.44 bar and $830 \mathrm{~K}$, respectively. The three lobed strut injectors plotted in Fig. 5 are investigated under these conditions. The inflow values of the air (at the nozzle throat) and hydrogen (at the nozzle exit) are summarized in Table II. Due to different nozzle areas of the struts, different hydrogen mass fluxes are injected. In case of strut I it is $6.46 \mathrm{~g} / \mathrm{s}$, in case of strut II $4.37 \mathrm{~g} / \mathrm{s}$, and strut III injects $3.41 \mathrm{~g} / \mathrm{s}$. The mass flow rate of the air is $332 \mathrm{~g} / \mathrm{s}$. Hence global equivalence ratios of $0.66,0.45$, and 0.35 are obtained for the struts I to III, respectively. Because the thermodynamic conditions are close to the self ignition limit of stoichiometric hydrogen/air mixtures the reaction mechanism is of great importance and detailed kinetic schemes are required for an accurate prediction of ignition delays. In Ref. [36] it is shown, that there is a high sensitivity of the ignition delay time to changes in temperature at temperature levels below $1000 \mathrm{~K}$ (at pressures of about 1 bar). Under such conditions reduced mechanisms (e.g. 7-species, 7-steps) are not able to accurately predict the lift-off height of the flame. Therefore the 9species, 20-step reaction mechanism of Jachimowski [31] has been chosen which is validated for such conditions. On the other hand detailed kinetic schemes cause long computational times being the reason why smaller grids with about 0.6 million volumes have been used for the reactive flow simulations. A grid refinement study has been performed for strut II using 3.4 million volumes. The differences obtained concerning the position of the flame and the species distributions have been small. Thus the use of the coarser mesh should have no effect on the outcome of this paper. The mixing efficiencies for the fine and 
coarse grid simulations are compared in Fig. 10. The distances of the first near wall cell centres to solid walls are between 1.2 and $2.3 \cdot 10^{-6} \mathrm{~m}$ resulting in $y^{+}$ values around 1 . Due to the fine near wall resolution cell aspect ratios of up to 1600 are obtained.

In case of axial fuel injection the chosen combustor inlet conditions allow both attached and detached flames. A diverging channel geometry (see Fig. 1) is required to compensate effects from heat release due to combustion. The geometric expansion causes an acceleration of the flow and decreases the air static temperature. If the expansion takes place upstream of the point of ignition it may cause a blow-off of the flame. For this reason the investigated combustor uses a first channel part (downstream of the strut) with a constant cross section to promote autoignition. According to the cold mixing investigations a length of $48 \mathrm{~mm}$ was chosen. After this length the upper and lower channel walls diverge at angles of $2.5^{\circ}$. A constant wall temperature of $700 \mathrm{~K}$ is assumed for all simulations.

\subsection{Mixing and fuel burnout}

Using the described combustor geometry and the inflow conditions of Table II, lifted flames are obtained for the three lobed strut injectors. Their lift-off lengths of about $50 \mathrm{~mm}$ (see Fig. 13 for strut III) are nearly identical. Figure 9 shows calculated distributions of oxygen (upper figures) and hydrogen (lower figures) mass fractions for channel cross sections $400 \mathrm{~mm}$ downstream of the injectors. At this position combustion should already be completed. In case of strut I the hydrogen nozzle covers the complete lobed shape of the strut including the vertical parts and the axises of rotation of the vortices. Along these axises of rotation high concentrations of hydrogen remain unburned due to a lack in oxygen caused by bad mixing. The same effect already has been observed for non-reactive flow. The oxygen which still is available near the upper and lower walls may not be transported fast enough into the combustion zone by turbulent diffusion. Even if a little bit better, the situation is similar for strut II. In case of strut III the injected hydrogen approximately matches the available oxygen in the core flow, which at $x=400 \mathrm{~mm}$ may be defined as the section up to $z= \pm 18 \mathrm{~mm}$ ). As may be seen from Fig. 9 the oxygen is nearly completely consumed in the core section for all three cases. Thus the reason for the unburned hydrogen in case of strut I and II is a mismatch between the injected hydrogen and the oxygen available in the core region. An important issue in the design of the strut injector is to create vortices which extend as far as possible in $z$-direction as to increase the area of oxygen supply for combustion. 
The problem of unburned hydrogen may be quantified by considering cross section averaged hydrogen mass fluxes or burning efficiencies. Figure 10 shows both performance parameters for the three struts along the channel length. Due to the ignition delay combustion starts about $50 \mathrm{~mm}$ downstream of the struts resulting in a strong decrease in hydrogen mass flux and a corresponding increase in burning efficiency. For all three cases the greatest parts of the consumed hydrogen is burned over a relatively short distance of about $20 \mathrm{~mm}$ (between $x=50$ and $70 \mathrm{~mm}$ ). This is because the ignition delay already allows a high degree of mixing and a partially premixed flame is obtained. After the initial fast combustion the burning efficiencies only increase slowly, indicating, that combustion is mixing dependent now and the burning dominated by turbulent diffusion. Figure 10 also quantifies the amount of unburned hydrogen. $450 \mathrm{~mm}$ downstream of the struts still $46 \%$ and $25 \%$ of the hydrogen is unburned for strut I and II, respectievely. In case of strut III only $12 \%$ of the injected hydrogen is left and nearly half of it is mixed with oxygen in a way that it could react under more favourable conditions. This may be seen from Fig. 11, where the mixing efficencies are plotted versus the channel length. The mixing efficiencies are increasing from the ends of the struts up to the points where ignition takes place (about $50 \mathrm{~mm}$ downstream). After ignition the initially fast combustion causes the mixing efficiencies to decrease strongly (the fraction of mixed hydrogen decreases). Further downstream (at $x>100 \mathrm{~mm}$ ) the mixing efficencies increase again indicating, that now the mixing process is faster than combustion. In case of strut III there is a significant rise in $\eta_{\text {mix }}$ up to a value of about 0.46 at $x=450 \mathrm{~mm}$. This means that from the $12 \%$ of the injected hydrogen being left, $46 \%$ are mixed with oxygen and are ready for combustion (if the temperature would be high enough and if there would be sufficient time). Thus strut III is relatively close to a complete burnout. Bad thermodynamic conditions (caused by the diverging channel geometry) are responsible that the hydrogen being mixed is not burned. This demonstrates that the geometric combustor design for low flight Mach numbers is a critical point. On one hand the static temperature has to be kept high enough to complete the fuel burnout and on the other hand thermal choking has to be avoided. If changing operating conditions are taken into account the situation is still more complex and a complete burnout at a stochiometric ratio probably reqires variable combustor geometries.

The low exit values in the mixing efficiencies for strut I and II indicate that there is little combustible mixture left. Reason is the mismatch between hydrogen and oxygen in the core region as explained before. At the end of the simulated combustor (450 mm downstream of the injector) $64 \%$ of the avialable oxygen is unburned in case of strut I, $66 \%$ in case of strut II and $69 \%$ in case of strut III, respectively.

Finally Fig. 12 shows normalized cross section averaged total pressures along the combustor length. The differences between the three the three struts are small. Despite the different amounts of hydrogen injected, the burned masses 
of hydrogen are quite similar. Reason is that the available oxygen in the main flow and not the hydrogen is limiting combustion. As a result the ignition delays, flame structures, and shock patterns are similar resulting in nearly identical losses in total pressure.

It may be concluded that the lobed strut injector is able to achieve a fast and complete mixing over a short length if the injected hydrogen matches the available oxygen in the region covered by the induced vortices. In a real scramjet combustor the air inflow conditions will change during flight and the injected hydrogen mass flux has to be changed correspondingly. This may be achieved by either changing the hydrogen injection conditions (e.g. pressure) or by changing the nozzle areas.

\subsection{Flame stabilization}

\section{Lifted flames}

Two stable modes of combustion have been observed numerically for the described lobed strut injectors and the given combustor geometry. For any mode the differences in the flow fields due to the different struts are small. Therefore results will be shown for strut III only. The two modes observed are related to lifted or attached flames. Using the inflow conditions given in Table II lifted flames are obtained (for all struts). Figure 13 shows temperature (upper figure) and Mach number (lower figure) distributions for the region downstream of the injector. There is a significant ignition delay and combustion starts about 50 mm downstream of the strut. As may be seen from the Mach number distribution the flame is stabilized by a subsonic bubble close to the point of ignition. The plotted white lines in the Mach number distribution indicate Mach 1 isolines and thus subsonic regions. The size of the subsonic part(s) vary in channel depth and they nearly disappear at some cross sections. The subsonic regions are located around the $x$-position, where the diverging channel part begins. Figure 14 shows a sketch of the physical processes taking place. In front of the subsonic bubble a small normal shock wave is formed (there are oblique shock waves further outside) which increases temperature and pressure and creates favourable conditions for ignition. In the present case temperature and pressure rise from about $800 \mathrm{~K}$ and 0.65 bar up to about $1250 \mathrm{~K}$ and 0.85 bar, respectively. This is sufficient for a fast ignition. Moreover, once the described shock structure and the subsonic bubble are formed, minor changes in inflow conditions do not effect combustion and autoignition. Da Silva et al. [37] observed a similar effect in a more theoretical two-dimensional study. In contrast to the present investigation, they used an oblique shock wave to artificially produce a subsonic zone where recirculation occurred. This is not the case in the present flow field where the Mach number decreases to Ma $\approx 0.6$ in the 
subsonic bubble but there is no recirculation. It is avoided by using a diverging channel geometry which causes a pressure drop. Due to the upwind influence in the subsonic region the pressure rise in the bubble is limited to maximum values of about 1 bar. Moreover, the flow acceleration in the diverging channel part also causes the subsonic bubble to disappear quickly. The advantage of such types of lifted kinetically stabilized flames is that combustion and thus high temperature regions are away from solid walls and from the strut. To extend the range of stability additional oblique shocks waves could be used (e.g. by ramps mounted to the upper and lower walls) similar to the concept of da Silva et al. [37].

\section{Attached flames}

More stable than the described lifted flames are combustion concepts, where the flame is attached to the strut. An increase in air temperature or in hydrogen injection pressure, a larger heat release due to combustion, or different shock structures at the end of the strut may cause the lifted flame to move upstream and directly anchor downstream of the strut. In the present investigation such an upstream movement of the flame is caused by an increased hydrogen injection pressure ( 0.854 bar instead of 0.8 bar). The increased pressure causes different pressure adaptation mechanisms by shock waves and expansion fans at the hydrogen nozzle exit as well as a higher hydrogen mass flux. The corresponding temperature and Mach number distributions are plotted in Fig. 15. Tiny subsonic zones with recirculation are formed behind the solid walls at the end of the strut. The white lines in the Mach number distribution of Fig. 15 indicate Mach 1 isolines. The subsonic region where the greatest part of combustion takes place is much larger now but still without recirculation. The length from the point of ignition up to the diverging channel part defines the length of the subsonic bubble. Again the subsonic regions disappear with begin of the diverging combustor part. Such subsonic regions are common for scramjet combustors at low flight Mach numbers.

\section{Thermal choking}

If the hydrogen mass flux is further increased above a certain limit, thermal choking occurs. Reason is that the heat release is too high for the first combustor part with a constant channel cross section. A normal shock wave stabilizes in front or at the tip of the strut and decelerates the complete combustor flow to subsonic speed. This has to be avoided.

\section{Conclusion}

A concept for mixing enhancement in turbulent high speed flows has been presented which is based on vorticity production by a favourable chosen strut 
geometry. The proposed lobed strut injector creates counter-rotating streamwise vortices into which the hydrogen is injected. It is shown that the length to achieve a perfect mixing is strongly reduced by this technique. Critical regions for hydrogen injection where found to be close to the axises of rotation of the vortices. Moreover, the total amount of hydrogen injected should match the available oxygen in the cross section covered by the vortices. Oxygen which is available available outside of this region (near the upper and lower walls) may not be transported fast enough into the combustion zone an leaves the combustor unburned. Thus the combustor height either should be adapted to the height of the vortices or additional fuel has to be added (e.g. by wall injection) in order to reach a stoichiometric ratio. In the second case a staged injection concept may be used with wall injectors located in the diverging combustor part.

For the chosen combustor and strut geometries two stable modes of combustion are observed for inflow conditions which correspond to low scramjet flight Mach numbers ( 7 - 8). They are associated with lifted or attached flames. Lifted flames are obtained if the air temperature and the hydrogen mass flux are low. Increasing one of these values the flame moves upstream and directly anchors downstream of the strut. In both cases the flames are stabilized by subsonic regions. Lifted flames will be obtained for conditions close to the self ignition limit. An interesting principle of flame stabilization is observed for such cases. A small normal shock wave in front of the lifted flame creates favourable conditions for autoignition and stabilizes the flame. Due to the interaction with the diverging channel geometry no recirculation occurs in the subsonic bubble.

\section{References}

[1] D. Glawe, M. Samimiy, A. Nejad, T. Cheng, Effects of nozzle geometry on parallel injection from base of an extended strut into supersonic flow, Aiaa paper 95-0522 (1995).

[2] D. Papamoschou, Ananlysis of partially mixed supersonic ejector, Journal of Propulsion and Power 12 (1996) 736-741.

[3] J. Belanger, H. Hornung, Transverse jet mixing and combustion experiments in hypervelocity flows, Journal of Propulsion and Power 12 (1996) 186-192.

[4] A. Gardner, K. Hannemann, J. Steelant, A. Paull, Ground testing of the hyshot supersonic combustion experiment in heg and comparison with flight data, Aiaa paper 2004-3345 (2004).

[5] T. Mitani, T. Kouchi, Flame structures and combustion efficiency computed for a mach 6 scramjet engine, Combustion and Flame 142 (2005) 187-196. 
[6] I. Waitz, F. Marble, E. Zukoski, Investigation of a contoured wall injector for hypervelocity mixing augmentation, AIAA Journal 31 (1993) 1014-1021.

[7] D. Riggins, Thrust losses in hypersonic engines, part 2: Applications, Journal of Propulsion and Power 13 (1997) 288-295.

[8] R. Baurle, R. Fuller, J. White, T. Chen, M. Gruber, A. Nejad, An investigation of advanced fuel injection schemes for scramjet combustion, Aiaa paper 98-0937 (1998).

[9] J. Strickland, T. Selerland, A. R. Karagozian, Numerical simulation of a lobed fuel injector, Journal of Propulsion and Power 10 (1998) 2950-2964.

[10] B. Charyulu, J. Kurian, P. Venugopalan, V. Sriamulu, Experimental study on mixing enhancement in two dimensional supersonic flow, Experiments in Fluids 24 (1998) 340-346.

[11] T. Sunami, M. Wendt, M. Nishioka, Supersonic mixing and combustion control using streamwise vorticity, Aiaa paper 98-3271 (1998).

[12] D. Riggins, C. McClinton, R. Rogers, R. Bittner, Investigation of scramjet injection strategies for high mach number flows, Journal of Propulsion and Power 11 (1995) 409-418.

[13] S. Cox, R. Fuller, J. Schetz, Vortical interactions generated by an injector array to enhance mixing in supersonic flow, Aiaa paper 94-0708 (1994).

[14] L. Maddalena, T. Campioli, J. Schetz, Experimental and computational investigation of light-gas injectors in mach 4.0 crossflow, Journal of Propulsion and Power 22 (2006) 1035-2006.

[15] T. Sunami, K. Itoh, T. K. nad K. Sato, Effects of streamwise vortices on scramjet combustion at mach 8-15 flight enthalpies - an experimental study in hiest, Isabe paper 2005-1028 (2005).

[16] E. Fernando, S. Menon, Mixing enhancement in compressible mixing layers: An experimental study, AIAA Journal 31 (1993) 278-285.

[17] J.-H. Kim, Y. Yoon, I.-S. Jeung, H. Huh, J.-Y. Choi, Numerical study of mixing enhancement by shock waves in model scramjet engine, AIAA Journal 41 (2003) 1074-1080.

[18] P. Gerlinger, P. Kasal, P. Stoll, D. Brüggemann, Experimental and numerical investigation of $2 \mathrm{~d}$ and $3 \mathrm{~d}$ parallel hydrogen/air mixing in supersonic flows, ISABE paper 2001-1019.

[19] M. Kodera, T. Sunami, K. Itoh, Numerical simulation of a scramjet engine for jaxa's flight experiment using hyshot, Aiaa paper 2005-3355 (Jan. 2005).

[20] T. Sunami, A. Murakami, M. Nishioka, Mixing and combustion control strategies for efficient scramjet operation in winde range of flight mach numbers, Aiaa paper 2002-5116 (2002). 
[21] P. Gerlinger, J. Algermissen, D. Brüggemann, Matrix dissipation for central difference schemes with combustion, AIAA Journal 33 (1995) 1865-1870.

[22] P. Gerlinger, P. Stoll, D. Brüggemann, An implicit multigrid method for the simulation of chemically reacting flows, Journal of Computational Physics 146 (1998) 322-345.

[23] P. Gerlinger, H. Möbus, D. Brüggemann, An implicit multigrid method for turbulent combustion, Journal of Computational Physics 167 (2001) 247-276.

[24] F. Schneider, P. Gerlinger, M. Aigner, 3d simulations of supersonic chemically reacting flows, in: E. Krause, W. Jäger, M. Resch (Eds.), High Performance Computing in Science and Engineering03, Springer Verlag, 2003, pp. 267-276.

[25] P. Gerlinger, M. Aigner, Multigrid simulations of detached shock waves, International Journal for Numerical Methods in Fluids 44 (2004) 1045-1061.

[26] P. Gerlinger, D. Brüggemann, Multigrid convergence acceleration for turbulent supersonic flows, International Journal for Numerical Methods in Fluids 24 (1997) 1019-1035.

[27] A. Jameson, S. Yoon, Lower-upper implicit schemes with multiple grids for the euler equations, AIAA Journal 25 (7) (1987) 929-935.

[28] J. Shuen, Upwind differencing and lu factorization for chemical non-equilibrium navier stokes equations, Journal of Computational Physics 99 (1992) 233-250.

[29] P. Stoll, P. Gerlinger, D. Brüggemann, Domain decomposition for an implicit lu-sgs scheme using overlapping grids, Aiaa paper 97-1869 (1997).

[30] T. Coakley, P. Huang, Turbulence modeling for high speed flows, Aiaa paper 92-0436 (1992).

[31] C. Jachimowski, An analytical study of the hydrogen-air reaction mechanism with application to scramjet combustion, Nasa-tp-2791 (1988).

[32] P. Kasal, P. Gerlinger, R. Walther, J. von Wolfersdorf, B. Weigand, Supersonic combustion: Fundamental investigation of aerothermodynamic key problems, Aiaa paper 2002-5119 (2002).

[33] P. Gerlinger, D. Brüggemann, Numerical investigation of hydrogen strut injections into supersonic airflows, Journal of Propulsion and Power 16 (2000) $22-28$.

[34] P. Gerlinger, P. Kasal, F. Schneider, J. von Wolfersdorf, M. Aigner, Experimental and numerical investigation of lobed strut injectors for supersonic combustion, in: D. Jacob, G. Sachs, S. Wagner (Eds.), Basic Research and Technologies for Two-Stage-to-Orbit Vehicles, Wiley-VCH Verlag GmbH \& Co. KGaG, 2005, pp. 365-382.

[35] S.-H. Lee, Characteristics of dual transverse injection in scramjet combustion, part 2: Combustion, Journal of Propulsion and Power 22 (5) (2006) 1020-1026. 
[36] P. Gerlinger, K. Nold, M. Aigner, Investigation of hydrogen/air reaction mechanisms for the simulation of supersonic combustion, submitted to Combustion and Flame.

[37] L. da Silva, V. Sabel'nikov, B. Deshaies, Stabilization of supersonic combustion by a free recirculating bubble: A numerical study, AIAA Journal 35 (1997) $1782-1784$. 
Table 1

Inflow conditions for air (at the nozzle throat) and hydrogen (at the strut injector exit) for the mixing investigations.

\begin{tabular}{lccc}
\hline & air & strut I & strut II \\
\hline$p(\mathrm{bar})$ & 2.11 & 0.55 & 0.55 \\
$u(\mathrm{~m} / \mathrm{s})$ & 320 & 1954 & 1954 \\
$T(\mathrm{~K})$ & 255 & 164 & 164 \\
$A_{\mathrm{H}_{2}}\left(\mathrm{~mm}^{2}\right)$ & - & 38.1 & 25.8 \\
$M a$ & 1 & 2 & 2 \\
$\dot{m}(\mathrm{~g} / \mathrm{s})$ & 740 & 6.01 & 4.07 \\
\hline
\end{tabular}

Table 2

Inflow conditions for air (at the nozzle throat) and hydrogen (at the strut injector exit) for the combustion investigations.

\begin{tabular}{lcccc}
\hline & air & strut I & strut II & strut III \\
\hline$p(\mathrm{bar})$ & 2.11 & 0.8 & 0.8 & 0.8 \\
$u(\mathrm{~m} / \mathrm{s})$ & 723 & 2643 & 2643 & 2643 \\
$T(\mathrm{~K})$ & 1300 & 300 & 300 & 300 \\
$A_{\mathrm{H}_{2}}\left(\mathrm{~mm}^{2}\right)$ & - & 38.1 & 25.8 & 22.4 \\
$M a$ & 1 & 2 & 2 & 2 \\
$\dot{m}(\mathrm{~g} / \mathrm{s})$ & 330 & 6.46 & 4.37 & 3.41 \\
\hline
\end{tabular}




\section{FIGURES}
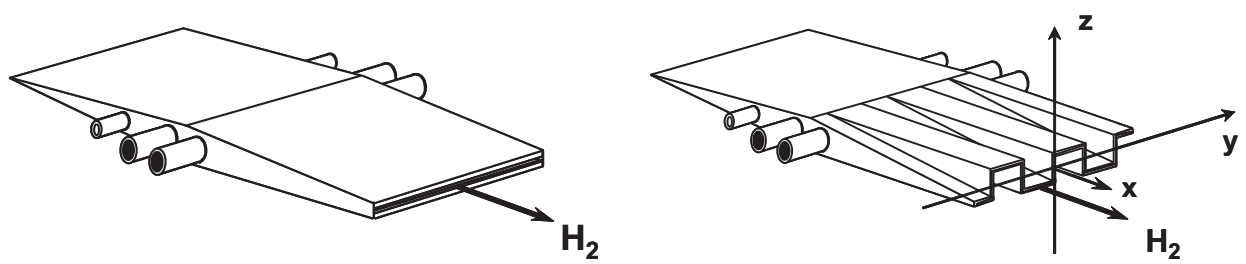

Fig. 1. Geometries of the planar and lobed strut injectors and coordinate directions.

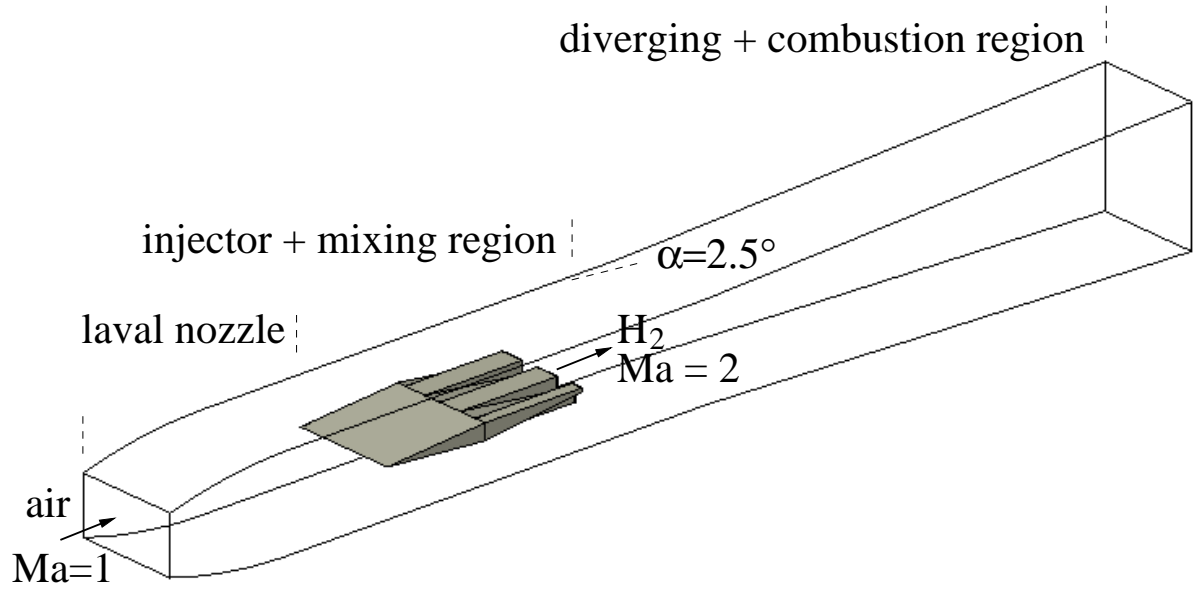

Fig. 2. Geometry of the model scramjet combustor with a lobed strut for fuel injection and a diverging channel section (in case of reactive flow). 


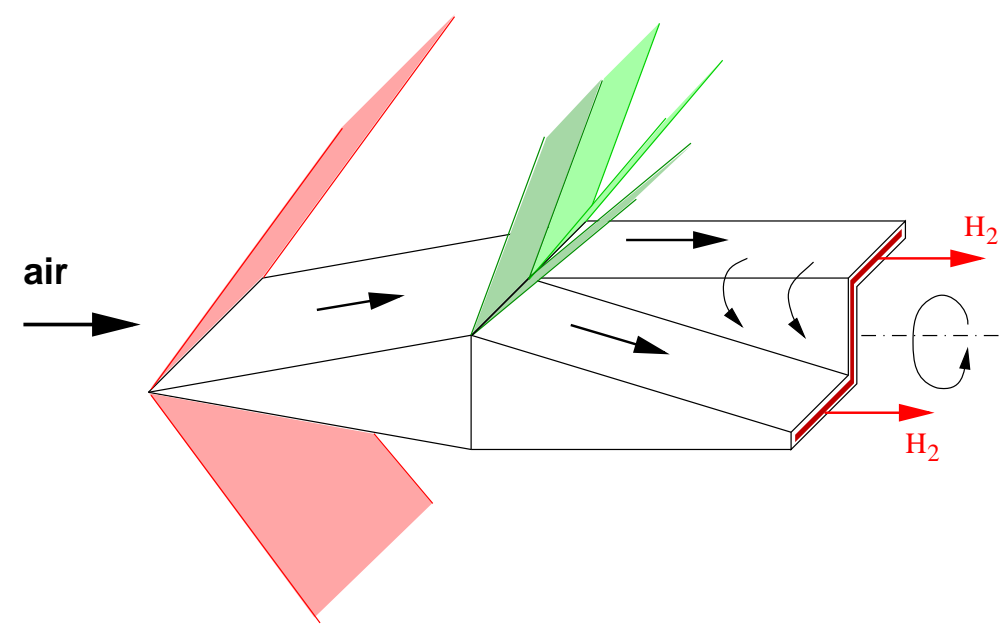

Fig. 3. Creation of streamwise vorticity by the lobed structure of the strut.

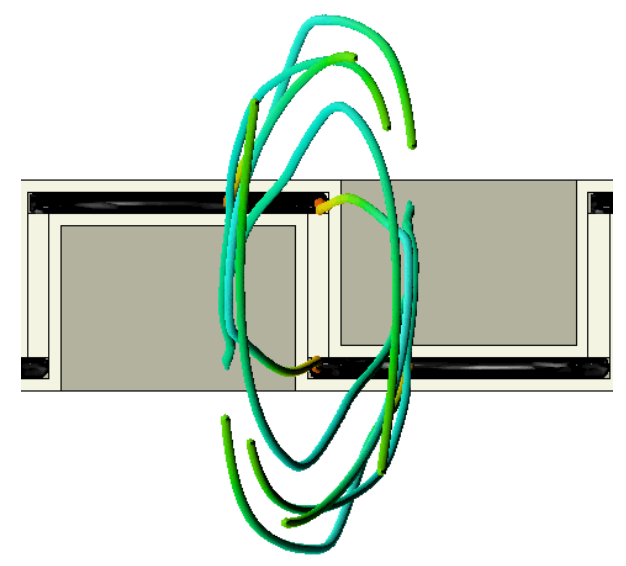

Fig. 4. Streamlines starting at the end of the strut in a view back from the channel exit. The streamlines are obtained for strut II from a simulation including combustion. 

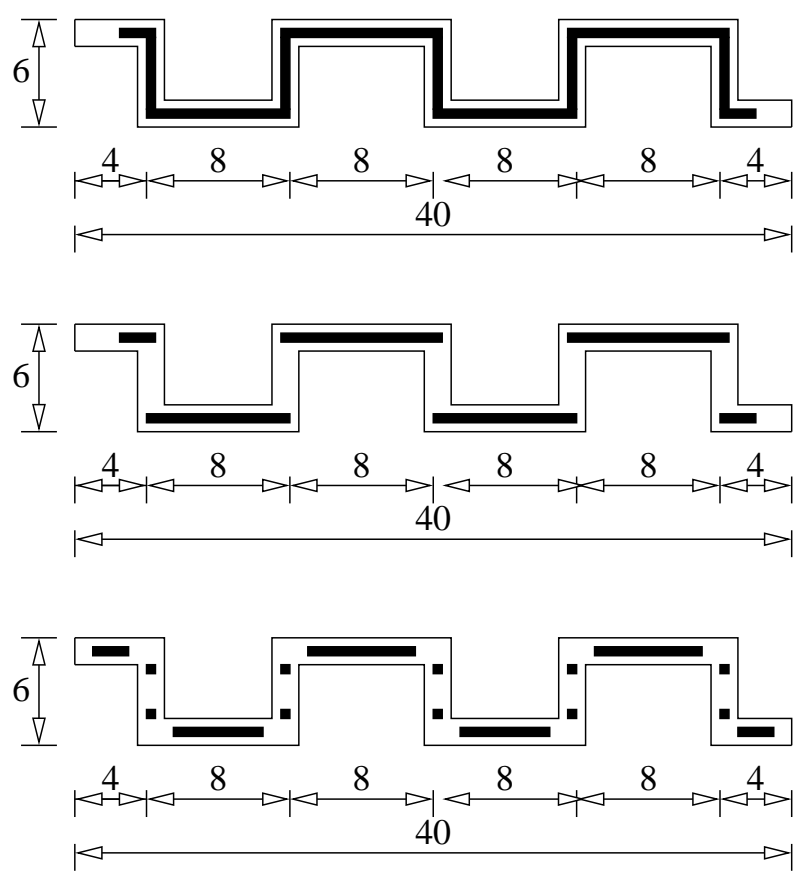

Fig. 5. Areas of hydrogen injection (black) for strut I to III from top to bottom (sizes in $\mathrm{mm}$ ). 

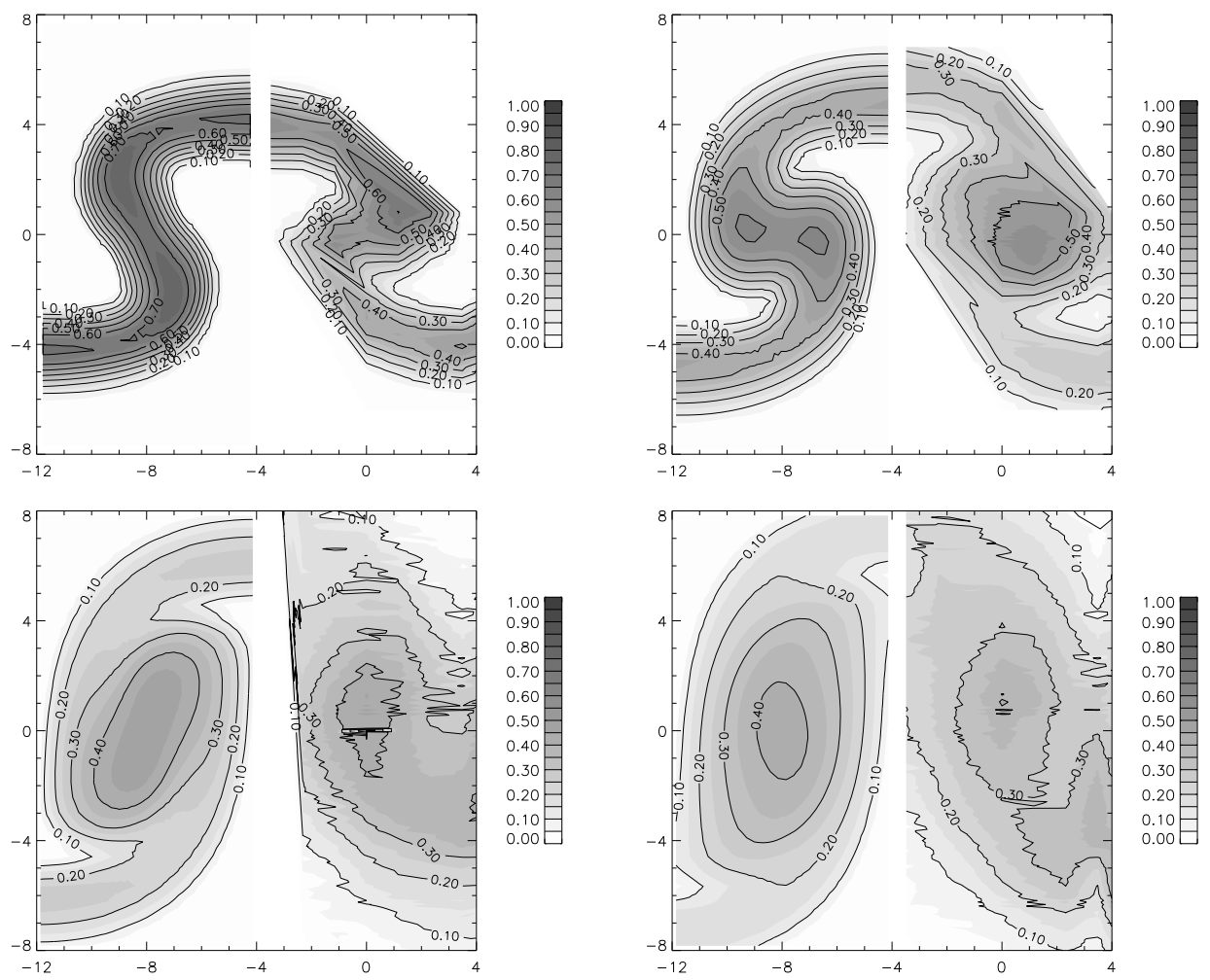

Fig. 6. Calculated (always left side) and measured (always right side) hydrogen molar fractions in $y$ - $z$ cross sections at $x=25,50,100$, and $150 \mathrm{~mm}$ downstream of the strut injector. 

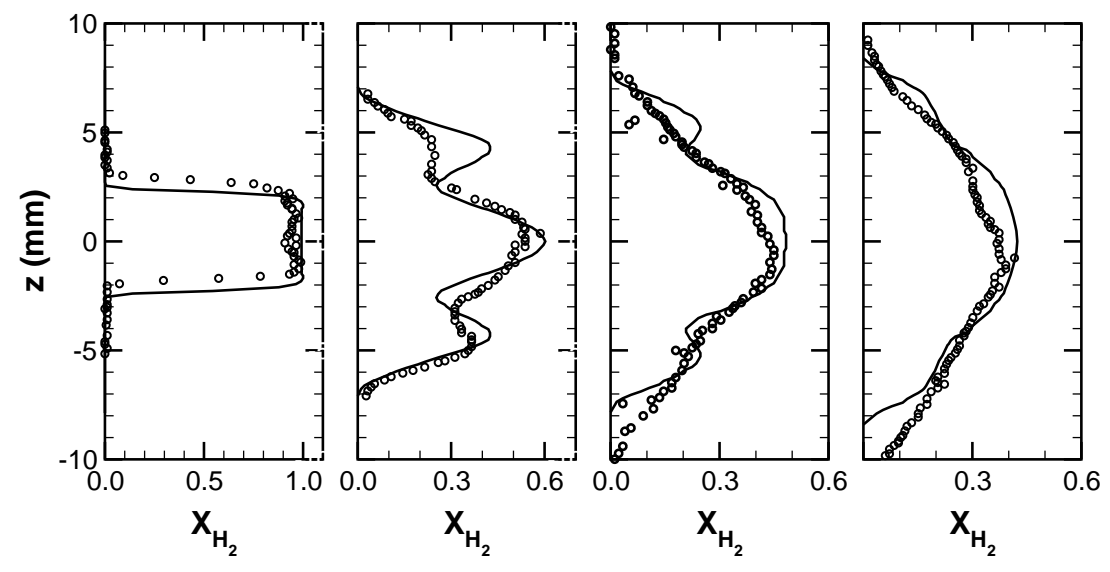

Fig. 7. Profiles of hydrogen molar fractions for $y=0 \mathrm{~mm}$ at $x=3,50,100$, and $150 \mathrm{~mm}$ downstream of the lobed strut I injector (solid lines simulation, symbols experiment).

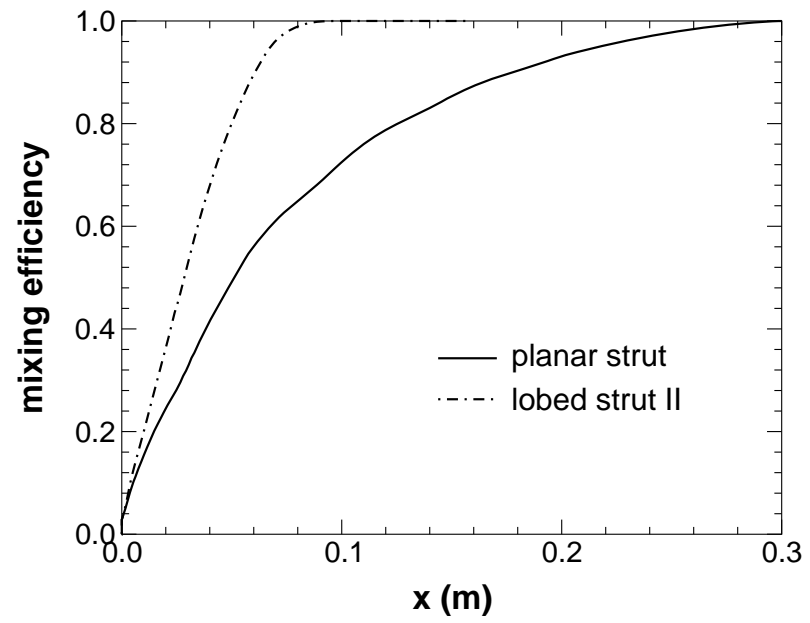

Fig. 8. Mixing efficiencies for the planar strut and lobed strut II. 

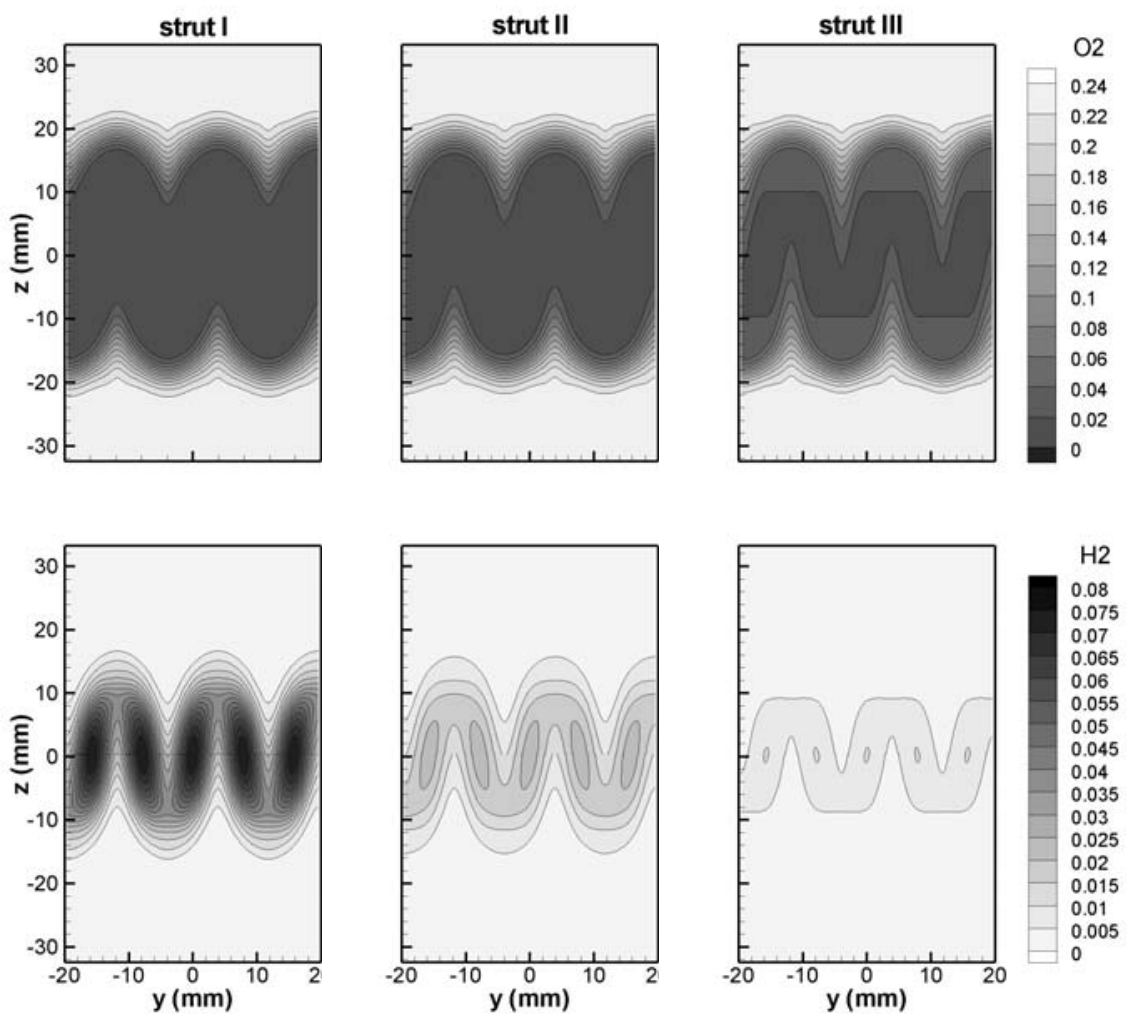

Fig. 9. Oxygen (upper figures) and hydrogen (lower figures) mass fraction distributions for strut I to III in cross sections $400 \mathrm{~mm}$ downstream of the injector (with combustion). 


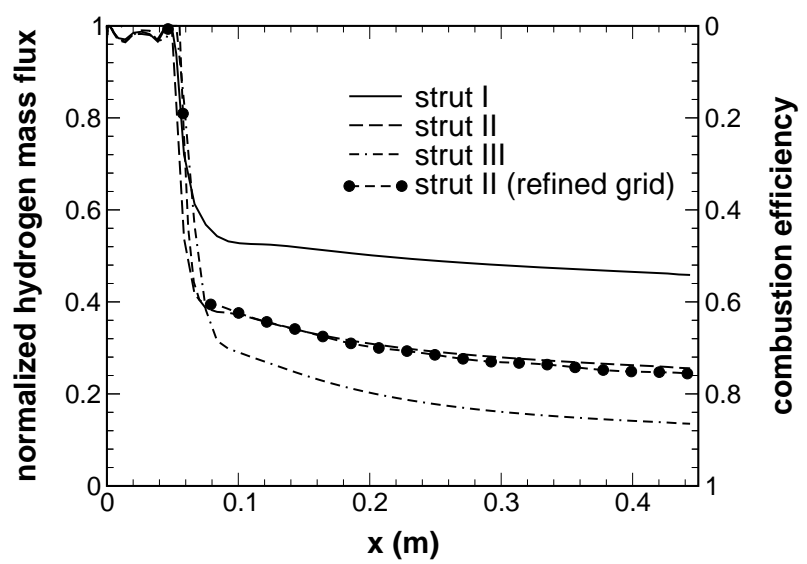

Fig. 10. Normalized hydrogen mass fluxes and burning efficiencies along the channel length using strut I to III (with combustion).

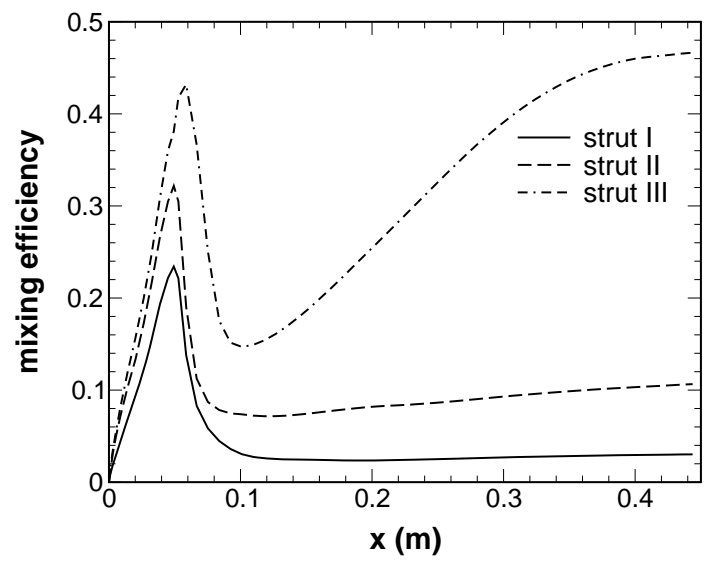

Fig. 11. Mixing efficiencies along the channel length using strut I to III (with combustion). 


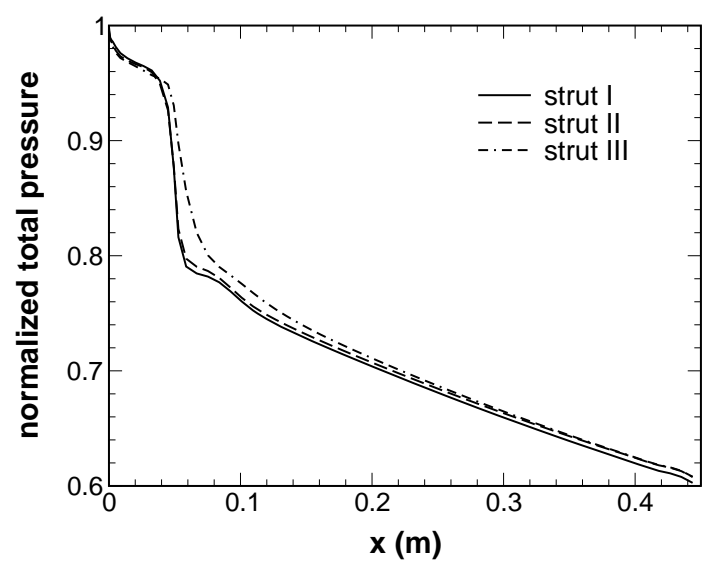

Fig. 12. Normalized mass flux averaged total pressure along the channel length using strut I to III (with combustion). 

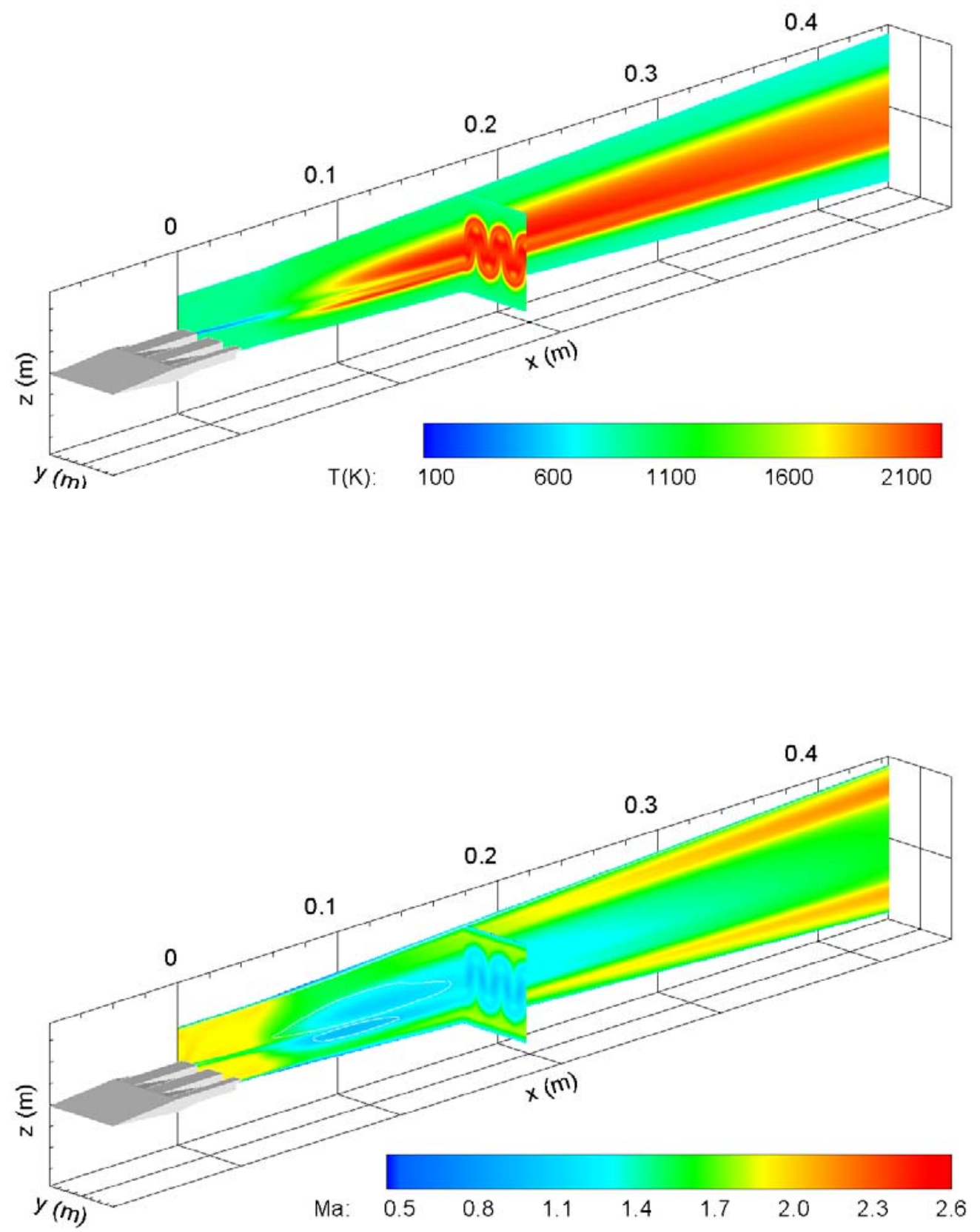

Fig. 13. Calculated temperature (upper figure) and Mach number (lower figure) distributions for strut III using the standard injection conditions of Table II. 


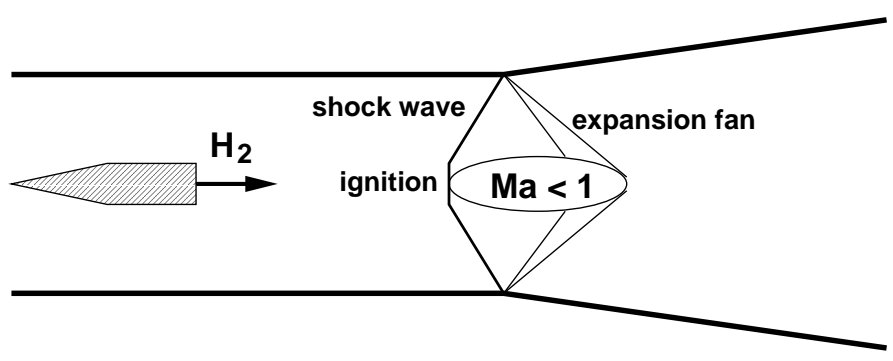

Fig. 14. Flame stabilization of a lifted flame by a subsonic bubble. 

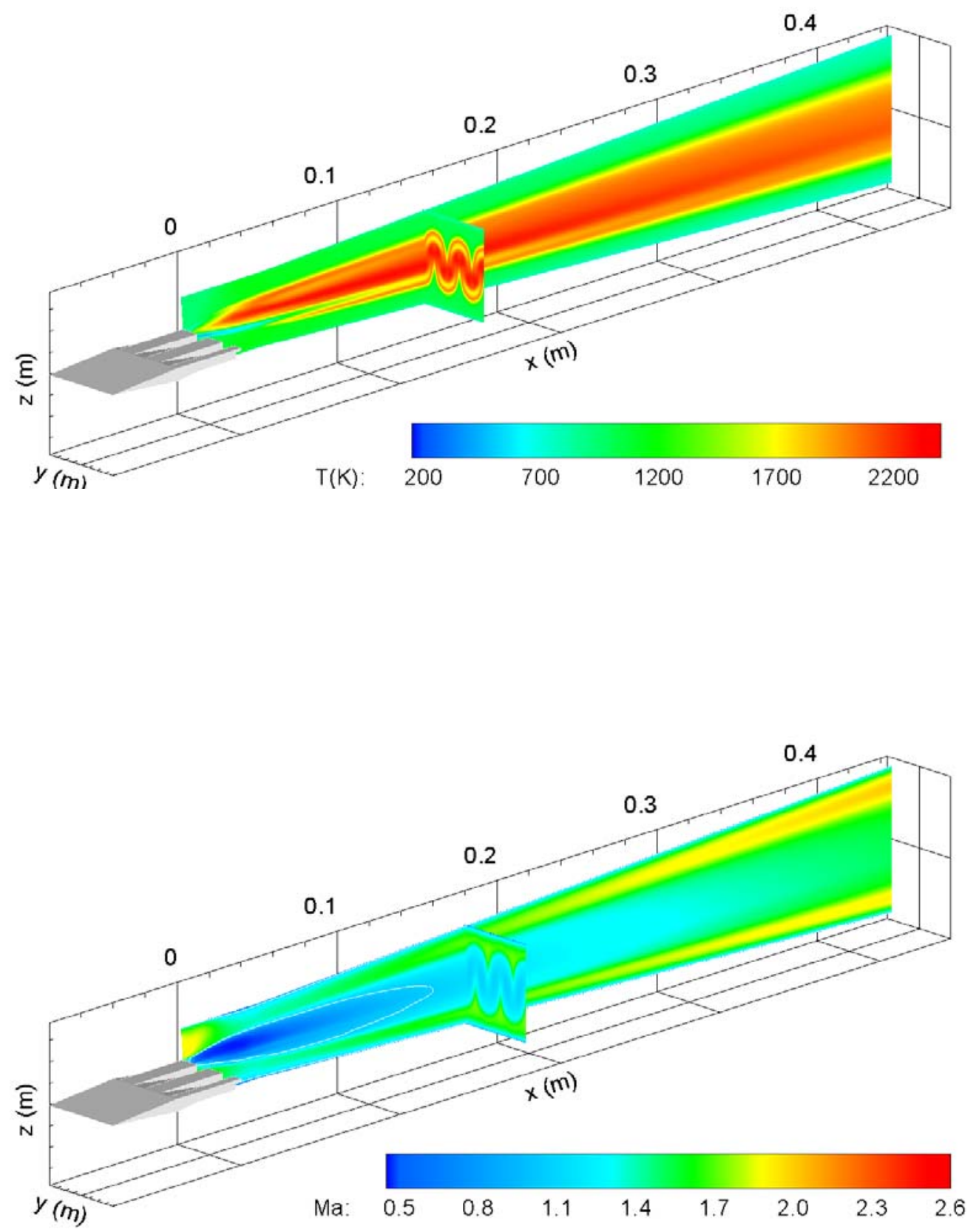

Fig. 15. Calculated temperature (upper figure) and Mach number (lower figure) distributions for strut III using the standard injection conditions of Table II with exception of an increased hydrogen pressure of $p=0.854$ bar. 